\title{
Neutrosophic Functions of the Joint Roughness Coefficient and the Shear Strength: A Case Study from the Pyroclastic Rock Mass in Shaoxing City, China
}

\author{
Jun Ye, Rui Yong, Qi-Feng Liang, Man Huang, and Shi-Gui Du \\ Key Laboratory of Rock Mechanics and Geohazards, Shaoxing University, Zhejiang, Shaoxing 312000, China \\ Correspondence should be addressed to Shi-Gui Du; dsg@usx.edu.cn
}

Received 29 February 2016; Accepted 26 April 2016

Academic Editor: Gregory Chagnon

Copyright @ 2016 Jun Ye et al. This is an open access article distributed under the Creative Commons Attribution License, which permits unrestricted use, distribution, and reproduction in any medium, provided the original work is properly cited.

Many studies have been carried out to investigate the scale effect on the shear behavior of rock joints. However, existing methods are difficult to determinate the joint roughness coefficient (JRC) and the shear strength of rock joints with incomplete and indeterminate information; the nature of scale dependency of rock joints is still unknown and remains an ongoing debate. Thus, this paper establishes two neutrosophic functions of the JRC values and the shear strength based on neutrosophic theory to express and handle the incomplete and indeterminate problems in the analyses of the JRC and the shear strength. An example, including four rock joint samples derived from the pyroclastic rock mass in Shaoxing city, China, is provided to show the effectiveness and rationality of the developed method. The experimental results demonstrate that the proposed neutrosophic functions can express and deal with the incomplete and indeterminate problems of the test data caused by geometry complexity of the rock joint surface and sampling bias. They provide a new approach for estimating the JRC values of the different-sized test profiles and the peak shear strength of rock joints.

\section{Introduction}

In mining, civil, hydraulic, and petroleum engineering, engineers often face problems associated with jointed rock mass [1]. One of the most challenging tasks in engineering rock mechanics is comprehensively understanding the characteristics of rock joints, including orientation, extent, planarity, roughness, and strength of wall rock asperities. Roughness refers to the local departures from planarity at both small and large scale [2] and has a direct impact on the shear strength of rock joints. It has been researched in the past four decades since Barton introduced the joint roughness coefficient (JRC) [3].

A variety of researches have been carried out to quantitatively describe the surface roughness of fractured surfaces in an attempt to relate the conventional geometric parameters or fractal dimensions of surface roughness to the mechanical behavior of rock joints [4]. The most widely used formula for estimating the shear strength was proposed by Barton [5] as follows:

$$
\tau=\sigma_{n} \tan \left[\mathrm{JRC} \log _{10}\left(\frac{\mathrm{JCS}}{\sigma_{n}}\right)+\phi_{b}\right]
$$

where $\tau$ is the peak shear strength of rock joints, $\sigma_{n}$ is the effective normal stress, $\phi_{b}$ is the basic friction angle of the discontinuity surface, and JCS is the joint wall compressive strength.

The variation in the mechanical behavior of joints with increasing scale is a well-known phenomenon which makes the mechanical parameters worked out in the laboratory unsuitable for the natural discontinuities located in real condition. To better understand the deformational behavior of rock systems, a large number of studies have been carried out over the last four decades for exploring the scale effect on the shear strength of rock joints [5-9]. However, many of these studies have produced conflicting results and the rock 
joints of the same lithology may exhibit an extremely large range in mechanical properties $[10,11]$. The uncertainties in test results are mainly caused by the complex properties of surface roughness of rock joints. Du [12] found that the rock joint roughness in nature generally has the appearance-like nonuniformity, anisotropy, inhomogeneity, and scale effect. To describe the complex structure of joint surface, Chen et al. [13] proposed a geological statistics method to analyze the anisotropy and the size effects of structural surface of rocks. Meanwhile, the sampling bias and sampling disturbance effects may be responsible for incorrect conclusions concerning some of the apparent scale effects [14]. Time and budget constraints make it unfeasible to obtain sufficient data by lots of field experiments. Due to the special limitation in measurement accuracy and the variability of roughness, it is difficult to find a certain equation for describing the scale effect of different-sized rock joint samples.

Neutrosophy means the study of ideas and notions which are not true, nor false, but its study is between true and false, that is, neutral, indeterminate, unclear, vague, ambiguous, incomplete, contradictory, and so forth. Many types of indeterminacies are contained in many real situations. Therefore, the neutrosophic logic, neutrosophic set, neutrosophic probability, neutrosophic statistics, neutrosophic measure, neutrosophic precalculus, neutrosophic calculus, and so forth were born in neutrosophy [15]. Neutrosophy theory is very suitable for describing the incomplete and indeterminate information. Since there is the incomplete and indeterminate information in the JRC values of different-sized samples and the shear strength of rock joints indicated in previous researches, neutrosophic function is helpful to better express these incomplete and indeterminate phenomena and can express the relationship of the scale effect of JRC and the shear strength of rock joints. To do so, we use neutrosophic functions to describe the scale effect of JRC and shear strength and verify the effectiveness and rationality of the neutrosophic functions of the JRC values and the shear strength by real testing data for four large-scale rock joint samples in the field.

The rest of the paper is organized as follows. In Section 2, we introduce the some concepts of neutrosophic functions. In Section 3, the neutrosophic functions of JRC and the shear strength are established in different sampling lengths. In Section 4, a practical example based on real testing data for four test samples is given to illustrate the application and effectiveness of the developed approach. Finally, some final remarks and further work are given in Section 5.

\section{Some Concepts of Neutrosophic Functions}

Smarandache [15] firstly proposed the neutrosophic functions and neutrosophic calculus to handle various problems of indeterminacy in real world.

Let us consider a neutrosophic function (thick function) $f: R \rightarrow F(R)$, and then

$$
f(x)=[3 x, 3 x+3] \text { for any } x \in R,
$$

where $R$ is all real numbers and $F(R)$ is the set of all subsets of $R$.

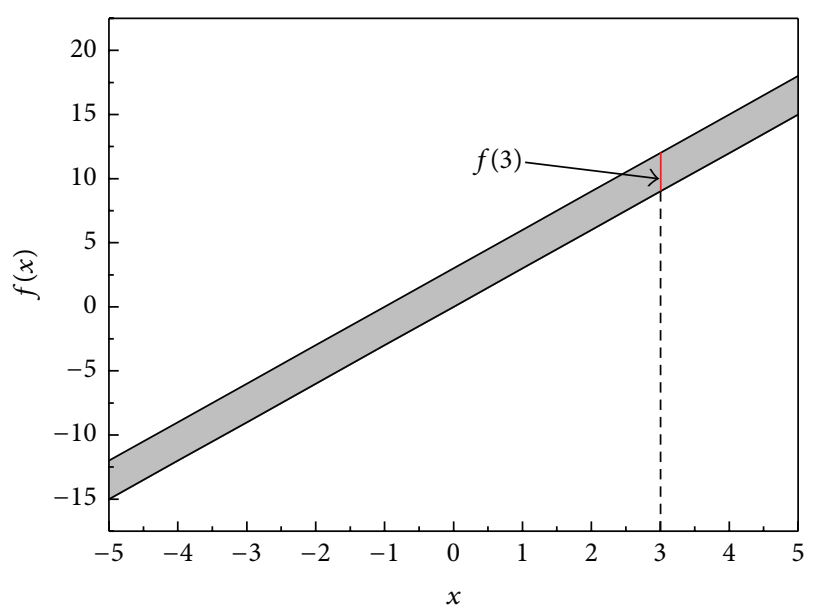

FIGURE 1: The graph of the neutrosophic function (thick function) $f(x)$.

Thus, its graph is shown in Figure 1.

For example, if $x=3, f(3)=[3 \times 3,3 \times 3+3]=[9$, $12]$, which is shown in Figure 1. The grey area indicates the indeterminate range between $f_{1}(x)=3 x$ and $f_{2}(x)=3 x+3$.

Let us consider another neutrosophic function (thick function) $g: R \rightarrow F(R)$; then

$$
g(x)=2 e^{[x, 2 x]}=\left[2 e^{x}, 2 e^{2 x}\right] \quad \text { for any } x \in R .
$$

If $x=2$, then $g(2)=\left[2 e^{2}, 2 e^{4}\right]$.

In general, we may define a neutrosophic function (thick function or interval function) $f: R \rightarrow F(R)$ as follows [15]:

$$
f(x)=\left[f_{1}(x), f_{2}(x)\right] .
$$

For example, if $x=1$, then $f(1)=\left[f_{1}(1), f_{2}(1)\right]$; the value of the neutrosophic function $f(x)$ is a vertical segment of line at $x=1$, which is the interval value $\left[f_{1}(1), f_{2}(1)\right]$.

However, we can also define an open interval function $f(x)=\left(f_{1}(x), f_{2}(x)\right)$ or semiopen/semiclosed interval functions $f(x)=\left[f_{1}(x), f_{2}(x)\right) / f(x)=\left(f_{1}(x), f_{2}(x)\right]$.

\section{Neutrosophic Functions of JRC and the Shear Strength}

As we know in rock mechanics, the joint roughness coefficient (JRC) of rock joints contains the indeterminacy in different sampling lengths and directions of rock joints [16] and then results in the indeterminacy of the shear strength of joints due to the corresponding relationship between JRC and the shear strength.

To study surface properties of the rock joints in Fusheng town, Shaoxing city, China, we measured 946 roughness profiles from the large, exposed pyroclastic rock mass by modified mechanical hand profilograph. The length of these samples ranged from $10 \mathrm{~cm}$ to $170 \mathrm{~cm}$. The images of these joint roughness profiles were obtained by large format scanner. The roughness profiles were digitized with the interval of $0.5 \mathrm{~mm}$ based on the grayscale image processing technique. 


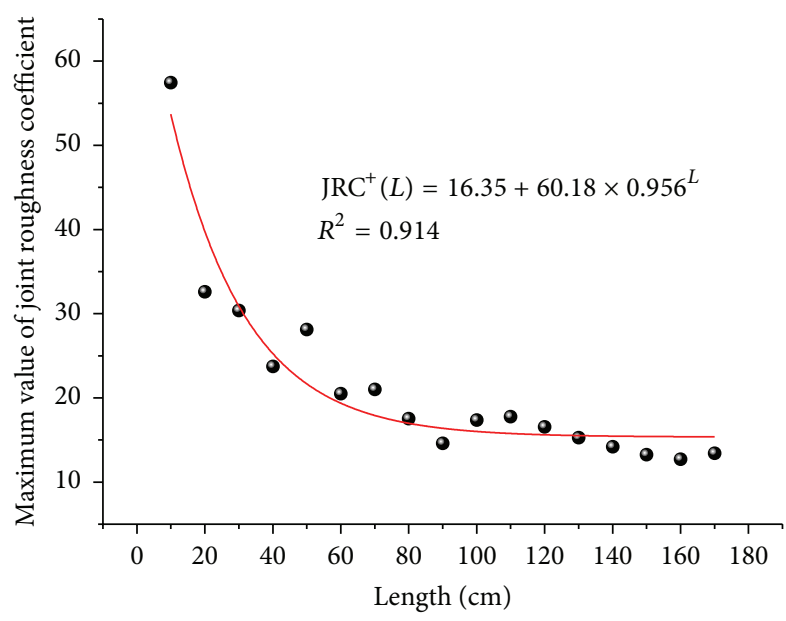

Figure 2: The correlation between the sampling length and maximum JRC values.

The JRC values were estimated based on the straight edge method [17]. The maximum and minimum JRC values of different-sized samples were obtained, respectively. Taking the maximum values as an example, Figure 2 shows the distribution of the maximum JRC values with the sample length. It denotes that the maximum values of JRC decrease gradually as the sampling length increases. An exponential function was proposed for describing their correlations as follows:

$$
\operatorname{JRC}^{+}(L)=16.35+60.18 \times 0.956^{L}
$$

for $L \in[10,170]$.

Similarly, the correlation between the sampling length and minimum JRC values was obtained as

$$
\operatorname{JRC}^{-}(L)=3.65+2.3 \times 0.958^{L} \quad \text { for } L \in[10,170] .
$$

Then the neutrosophic function for calculating the JRC values was proposed as the following formula:

$$
\begin{aligned}
\operatorname{JRC} & (L)=\left[\operatorname{JRC}^{-}(L), \operatorname{JRC}^{+}(L)\right] \\
& =\left[3.65+2.3 \times 0.958^{L}, 16.35+60.18 \times 0.956^{L}\right]
\end{aligned}
$$

for $L \in[10,170]$,

where $L$ is the sampling length and $\operatorname{JRC}^{-}(L)$ and $\operatorname{JRC}^{+}(L)$ are the lower and upper limits of the neutrosophic function $\operatorname{JRC}(L)$. Hence, the graph of $\operatorname{JRC}(L)$ is shown in Figure 2.

For example, if $L=80 \mathrm{~cm}$, then $\operatorname{JRC}(80)=[3.7243$, 17.9947], which is shown in Figure 3.

Based on the Barton shear strength [5], we propose the following neutrosophic function of shear strength:

$$
\begin{aligned}
\tau(\mathrm{JRC}) & =\left[\sigma_{n}^{-}, \sigma_{n}^{+}\right] \\
\cdot \tan [ & {\left[\operatorname{JRC}^{-}(L), \mathrm{JRC}^{-}(L)\right] \log _{10}\left(\frac{\left[\mathrm{JCS}^{-}, \mathrm{JCS}^{+}\right]}{\left[\sigma_{n}^{-}, \sigma_{n}^{+}\right]}\right) }
\end{aligned}
$$

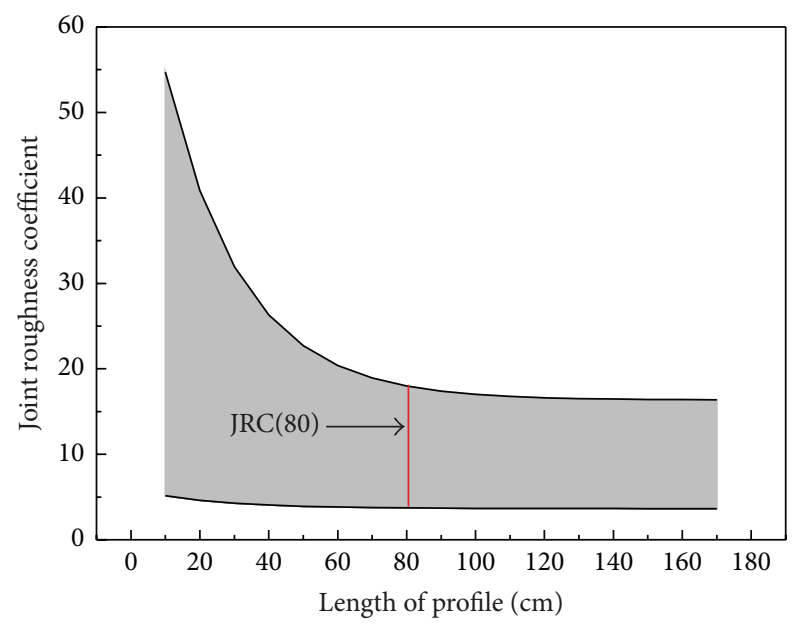

FIGURE 3: The graph of the neutrosophic function (thick function) $\operatorname{JRC}(L)$ for $L \in[10,170]$.

$$
\begin{aligned}
& \left.+\phi_{b}\right]=\left[\sigma_{n}^{-} \tan \left(\mathrm{JRC}^{-}(L) \log _{10}\left(\frac{\mathrm{JCS}^{-}}{\sigma_{n}^{+}}\right)+\phi_{b}\right),\right. \\
& \left.\sigma_{n}^{+} \tan \left(\mathrm{JRC}^{+}(L) \log _{10}\left(\frac{\mathrm{JCS}^{+}}{\sigma_{n}^{-}}\right)+\phi_{b}\right)\right],
\end{aligned}
$$

where $\tau(J R C)$ is the neutrosophic function of the peak shear strength of rock joints; $\sigma_{n}^{-}$and $\sigma_{n}^{+}$are the lower and upper limits of the effective normal stress; $\mathrm{JRC}^{-}(L)$ and $\mathrm{JRC}^{+}(L)$ are the lower and upper limits of the neutrosophic function $\operatorname{JRC}(L) ; \phi_{b}$ is the basic friction angle of the discontinuity surface; and JCS ${ }^{-}$and $\mathrm{JCS}^{+}$are the lower and upper limits of the joint wall compressive strength.

Obviously, (7) and (8) are two interval functions (indeterminate functions) and their indeterminate values lie within the intervals $\left[\mathrm{JRC}^{-}(L), \mathrm{JRC}^{+}(L)\right]$ and $\left[\tau^{-}(\mathrm{JRC}), \tau^{+}(\mathrm{JRC})\right]$, respectively. Hence, they can effectively describe the indeterminate phenomena of rock joints.

\section{Experimental Results and Discussion}

To demonstrate the effectiveness of the proposed neutrosophic functions, the samples of natural rock joints were selected to evaluate the scale effect of surface roughness in this study. We measured these testing samples from the pyroclastic rock mass that crop out at the east of Shaoxing City in Zhejiang Province, China (Figure 4). The pyroclastic rocks are volcaniclastic rocks formed by accumulation of pyroclasts (fragments) during Mesozoic explosive eruption by Yanshan movement. According to the size and abundance of their pyroclasts, these pyroclastic rocks are classified to be the lapillistones (pyroclasts $2-64 \mathrm{~mm}$ in size).

Generally, the rock joints play an important role in mechanical properties and deformation behavior of rock masses, the shear resistance hinges on the frictional resistance, and geometric irregularities along rock joint surfaces. In this study, the rock masses are highly fractured due to 


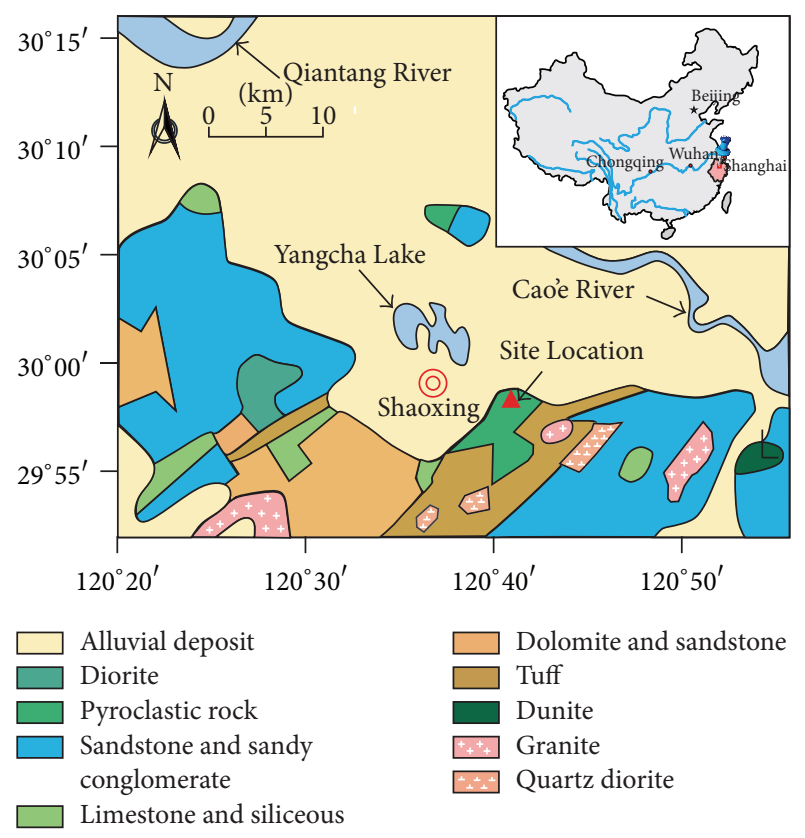

FIGURE 4: Location of the test site in simplified geological map (GPS: $\mathrm{N} 29^{\circ} 57^{\prime}$, E $120^{\circ} 43^{\prime}$ ).

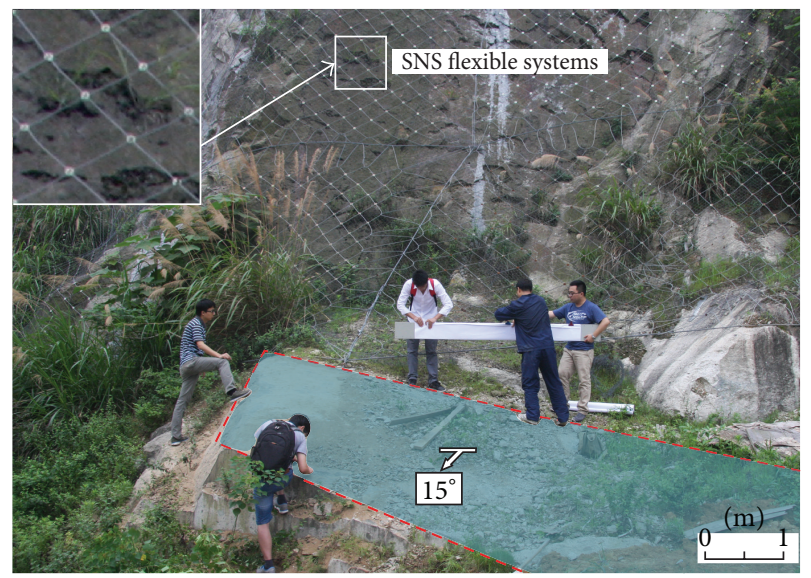

FIGURE 5: Photograph showing the fractured rock mass and the measurement area of rock joint set.

the mechanical behavior of these soft rocks. Three dominant joint sets are widely developed in this study area. The safety netting system (SNS) (diamond-shaped steel wire mesh) has been applied for protecting the potential rock slope or rock falls (Figure 5). Fifteen profiles of rock joint set (dip $15^{\circ}$, dip direction $95^{\circ}$ ) were measured in situ using modified mechanical hand profilograph $[18,19]$. The length of measured samples ranges from $172.69 \mathrm{~cm}$ to $223.94 \mathrm{~cm}$.

In the field, the length of the rock joint surface of interest is generally much longer than small-scale laboratory specimen, so JRC value must be estimated for full-scale surface. Barton and Bandis [20] proposed an alternative and simple method for estimating field-scale JRC, as shown in Figure 6. The approximate value of field-scale JRC is approximately determined from the measured values of amplitudes
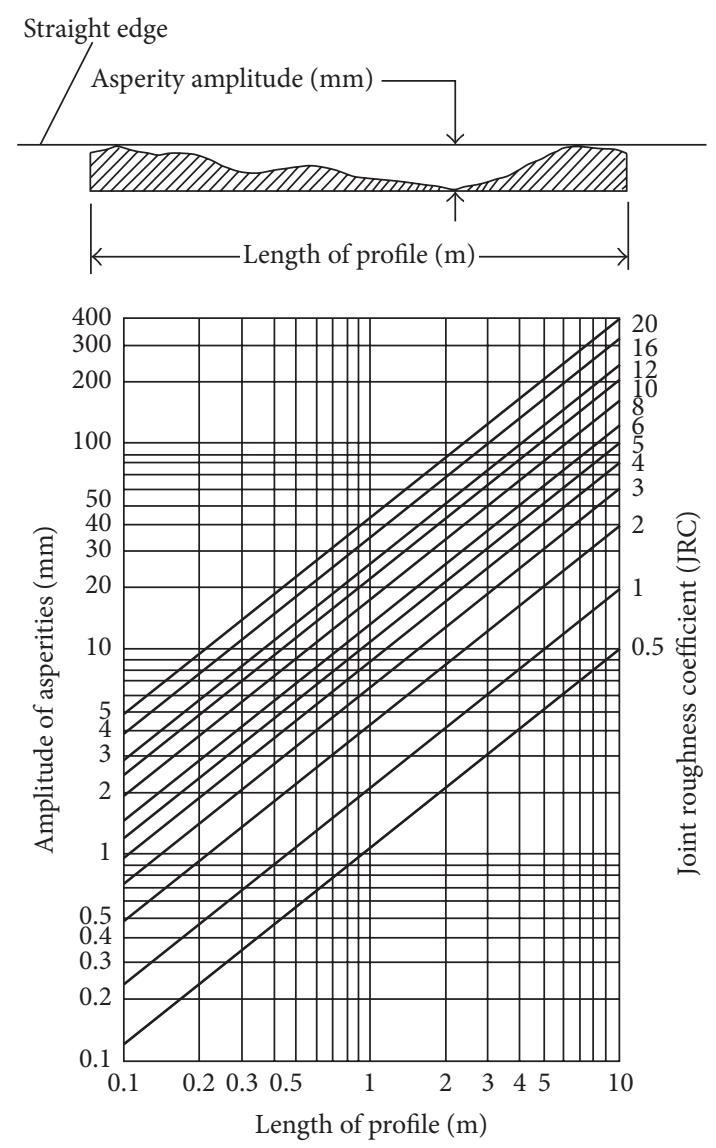

FIGURE 6: Estimating JRC from measurements of surface roughness amplitude from a straight edge [17].

and lengths of joint profiles. In this study, these roughness profiles were digitized in the sampling interval of $0.5 \mathrm{~mm}$, which we then used to determine JRC values. Firstly, each roughness profile was divided into small sized samples, in the length of $10 \mathrm{~cm}, 20 \mathrm{~cm}, 30 \mathrm{~cm}, 40 \mathrm{~cm}, 50 \mathrm{~cm}, 60 \mathrm{~cm}, 70 \mathrm{~cm}$, $80 \mathrm{~cm}, 90 \mathrm{~cm}, 100 \mathrm{~cm}, 110 \mathrm{~cm}, 120 \mathrm{~cm}, 130 \mathrm{~cm}, 140 \mathrm{~cm}, 150 \mathrm{~cm}$, $160 \mathrm{~cm}$, and $170 \mathrm{~cm}$. Note that the left side of the original rock joint profile along the potential sliding direction was chosen as the testing sample if the sample size is longer than halflength of the original profile. When we take the $176.91 \mathrm{~cm}$ original roughness profile as an example, there are 17 samples of $10 \mathrm{~cm}$ long, 8 samples of $20 \mathrm{~cm}$ long, 5 samples of $30 \mathrm{~cm}$ long, 4 samples of $40 \mathrm{~cm}$ long, 3 samples of $50 \mathrm{~cm}$ long, and two for each $60 \mathrm{~cm}, 70 \mathrm{~cm}$, and $80 \mathrm{~cm}$ sized samples; there is just one sample when the profile length is over $90 \mathrm{~cm}$.

Here, original roughness profiles (J1, J2, J3, and J4) were provided as an illustrative example and applied for analyzing the relationship between the joint roughness coefficient and their sample sizes, as shown in Figure 7. The test data and mean values of JRC for the various sizes of the rock joint samples J1, J2, J3, and J4 are illustrated in Tables 1-4, and then the graphs between the JRC values and the various sizes of the rock joint samples are shown in Figure 8. Clearly, the mean values of JRC of different-sized samples are decreased with the sample length increasing. 
TABLE 1: The test data and mean value of JRC of J1.

\begin{tabular}{llr}
\hline Length $(\mathrm{cm})$ & Test data of JRC & Mean value \\
\hline 10 & $13.608,8.196,14.842,15.747,15.478,10.121,24.452,20.134,11.879,12.280,15.029,8.156,8.374,19.908,19.160$, & 15.722 \\
20 & $34.872,15.034$ & 13.164 \\
30 & $8.884,15.083,8.791,22.140,10.292,9.787,12.900,17.436$ & 11.342 \\
40 & $8.364,7.924,19.591,8.385,12.445$ & 10.451 \\
50 & $8.172,13.304,9.072,11.256$ & 10.602 \\
60 & $12.987,12.770,4.456$ & 9.506 \\
70 & $8.581,10.430$ & 9.801 \\
80 & $9.473,10.129$ & 7.737 \\
90 & $8.486,6.988$ & 9.169 \\
100 & 9.169 & 9.263 \\
110 & 9.263 & 9.261 \\
120 & 9.261 & 8.490 \\
130 & 8.490 & 7.837 \\
140 & 7.837 & 7.277 \\
150 & 7.277 & 6.791 \\
160 & 6.791 & 6.746 \\
170 & 6.746 & 7.704 \\
\hline
\end{tabular}
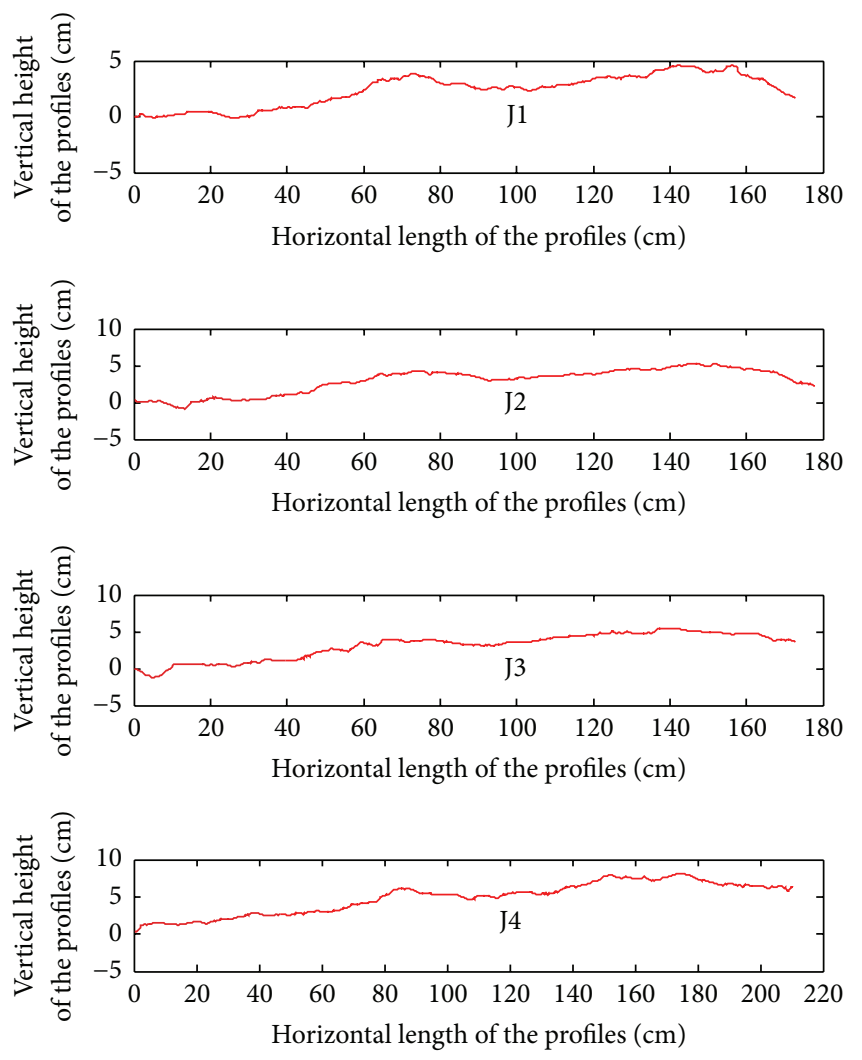

FIGURE 7: Digitization of original roughness profiles.

Although the difference between maximum JRC value and the minimum JRC value for $10 \mathrm{~cm}$ long samples (small samples) is the biggest of all original roughness profiles (J1, J2,
$\mathrm{J} 3$, and J4), we can see from Figure 8 that the JRC values of the test data fall in the interval value of the neutrosophic function (thick function) $\operatorname{JRC}(L)$ for $L$. Clearly, the neutrosophic function $\operatorname{JRC}(L)$ can indicate the range of the JRC values and show its effectiveness in the JRC analysis between the joint roughness coefficient and their sample sizes. Thus, it will provide an analysis foundation in order to predict the shear strength of rock joints.

To analyze the shear strength of rock joints, let us consider that the basic friction angle $\phi_{b}$ is $21^{\circ}, \sigma_{n}^{-}$is $1 \mathrm{MPa}$, and $\sigma_{n}^{+}$ is $2 \mathrm{MPa}$. The joints are completely unweathered; then JCS will be equal to the unconfined compression strength of the unweathered rock $\sigma_{c}$ [5]. The upper limit of the joint wall compressive strength $\mathrm{JCS}^{+}$is equal to $\sigma_{c}=10 \mathrm{MPa}$ and the lower limit JCS ${ }^{-}$is equal to $0.5 \sigma_{c}=5 \mathrm{MPa}$.

According to the neutrosophic function of shear strength in formula (6), the peak shear strength of rock joints can be predicted by the following formula:

$$
\begin{gathered}
\tau(L)=\left[\tan \left(\left(3.65+2.3 \times 0.958^{L}\right) \log _{10}\left(\frac{5}{2}\right)+21\right), 2\right. \\
\left.\cdot \tan \left(\left(16.35+60.18 \times 0.956^{L}\right) \log _{10}\left(\frac{10}{1}\right)+21\right)\right] .
\end{gathered}
$$

Then, based on (9), we can give the graph of the neutrosophic function $\tau(L)$ for $L \in[10,170]$, as shown in Figure 9 . The grey area in Figure 9 is its possible range (indeterminate area) of the shear strength of rock joints.

To predict the possible range of the shear strength of rock joints, we consider the following example.

Example 1. If $L=80 \mathrm{~cm}$, then $\tau(80)=[0.414,1.619](\mathrm{MPa})$, which is shown in Figure 9. Obviously, the possible value of the shear strength of rock joints for $L=80 \mathrm{~cm}$ may be within 
TABLE 2: The test data and mean value of JRC of J2.

\begin{tabular}{llr}
\hline Length $(\mathrm{cm})$ & Test data of JRC & Mean value \\
\hline 10 & $19.388,30.850,9.538,10.764,24.210,8.971,22.201,17.600,7.785,18.691,9.024,10.227,9.925,11.114,19.033$, & 15.261 \\
20 & $17.454,12.663$ & 13.398 \\
30 & $25.400,11.854,14.536,14.643,12.103,5.621,9.347,13.679$ & 11.689 \\
40 & $18.169,10.002,14.905,7.942,7.4270$ & 11.624 \\
50 & $13.627,10.858,10.810,11.202$ & 10.071 \\
60 & $12.987,12.770,4.456$ & 10.061 \\
70 & $11.016,9.105$ & 9.276 \\
80 & $10.157,8.394$ & 7.858 \\
90 & $8.887,6.830$ & 8.130 \\
100 & 8.130 & 9.392 \\
110 & 9.392 & 8.700 \\
120 & 8.700 & 8.290 \\
130 & 8.290 & 7.746 \\
140 & 7.746 & 7.342 \\
150 & 7.342 & 6.878 \\
160 & 6.878 & 7.351 \\
170 & 7.351 & 8.007 \\
\hline
\end{tabular}

TABLE 3: The test data and mean value of JRC of J3.

\begin{tabular}{llr}
\hline Length $(\mathrm{cm})$ & Test data of JRC & Mean value \\
\hline 10 & $57.464,13.48,18.888,17.990,17.595,37.676,28.627,12.708,8.585,13.930,10.742,8.063,18.024,18.868,7.812$, & 18.744 \\
20 & $9.189,19.002$ & 14.345 \\
30 & $31.402,11.898,18.838,15.541,11.540,6.391,12.870,6.283$ & 12.070 \\
40 & $20.935,13.115,11.630,4.936,9.732$ & 11.811 \\
50 & $15.701,13.389,9.451,8.704$ & 10.540 \\
60 & $12.561,11.487,7.572$ & 9.282 \\
70 & $10.744,7.821$ & 8.254 \\
80 & $9.209,7.298$ & 7.120 \\
90 & $8.0578,6.182$ & 9.158 \\
100 & 9.158 & 9.135 \\
110 & 9.135 & 8.304 \\
120 & 8.304 & 7.612 \\
130 & 7.612 & 7.043 \\
140 & 7.043 & 7.009 \\
150 & 7.009 & 7.211 \\
160 & 7.211 & 7.265 \\
170 & 7.265 & 7.955 \\
\hline
\end{tabular}

the interval $[0.414,1.619](\mathrm{MPa})$. This result may provide a theoretical analysis method for predicting the shear strength of rock joints in the specific district in Shaoxing city.

From the above analyses, we can see that the proposed neutrosophic functions can estimate the JRC values and the shear strength of rock joints and demostrate their effectiveness in the specific district. Therefore, the advantage is that neutrosophic functions can effectively express and analyze these inconplete and indeterminate problems because existing methods cannot express and handle these incomplete and indeterminate problems.

\section{Conclusion}

JRC is one of the most important parameters utilized in calculating shear strength of rough joints in rock masses. However, 


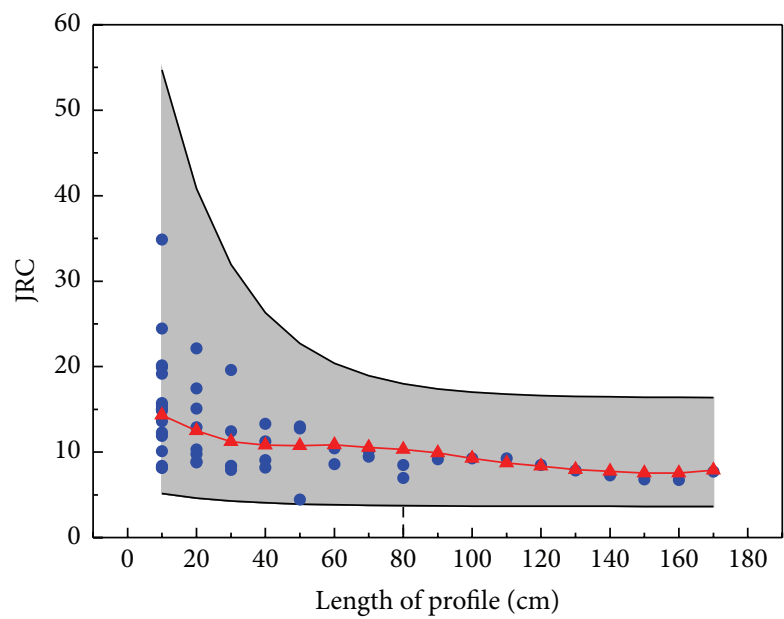

- Test data

$-\Delta-$ Mean value

(a)

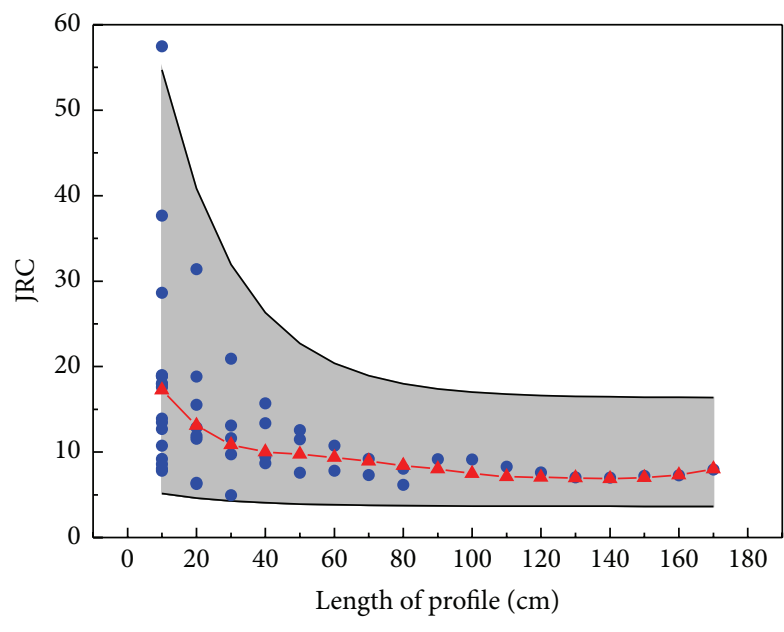

- Test data

-^- Mean value

(c)

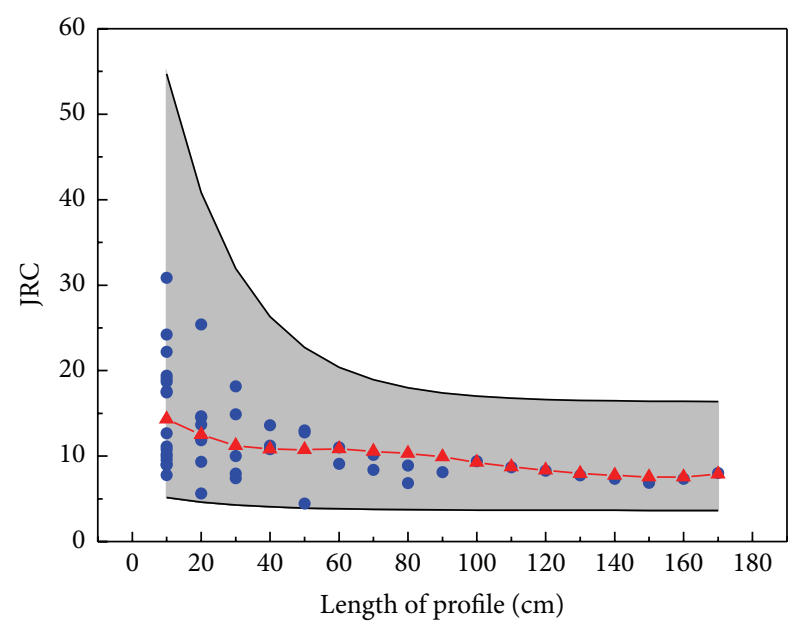

- Test data

$-\Delta-$ Mean value

(b)

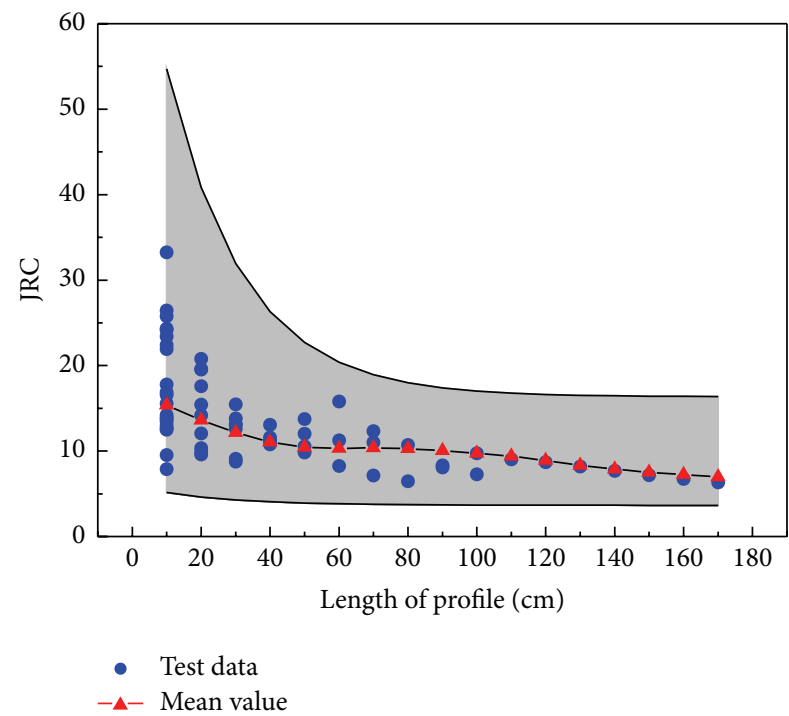

(d)

FIgURE 8: The relationship between the JRC values and the sizes of the rock joint samples. (a) J1; (b) J2; (c) J3; and (d) J4.

the incomplete and indeterminate problems generally exist in the scale effect test of rock joints, due to the geometry complexity of the rock joint surface and sampling bias. A quantitative description of roughness, suitable for incorporation into theoretically based models of joint behavior, should be proposed to express and deal with the incomplete and indeterminate problems of the JRC and shear strength of rough joints. This paper developed the two neutrosophic functions for describing the scale effect of JRC values and the shear strength of rock joints. They may provide a theoretical analysis method for predicting the JRC values and the shear strength of rock joints in the specific district in Shaoxing city.

By analyzing real test data for the four samples, the JRC values of the different-sized test profiles have greater discreteness in small samples than those in large samples.
Then, the mean values of JRC are declined with the sample length increasing. The results of the example showed that all the JRC values fall in the interval values of the neutrosophic function of JRC. Hence, the proposed neutrosophic function of JRC demonstrated its effectiveness in estimating the JRC values. On the basis of the proposed neutrosophic function of the peak shear strength of rock joints, an example was provided to show the effectiveness of a theoretical analysis method for the shear strength of rock joints in the specific district in Shaoxing city.

The proposed neutrosophic functions can express and deal with the incomplete and indeterminate problems of the test data, which provide a new way for the analyses of the JRC values of the different-sized test profiles and the peak shear strength of rock joints. In the future, we will further develop 
TABLE 4: The test data and mean value of JRC of J4.

\begin{tabular}{llr}
\hline Length $(\mathrm{cm})$ & Test data of JRC & Mean value \\
\hline 10 & $33.265,13.451,16.894,7.898,12.925,9.519,17.780,23.429,26.439,13.870,24.149,21.935,13.749,12.593,16.588$, & 18.058 \\
& $15.549,22.397,25.772,14.201,12.490,24.323$ & 14.895 \\
20 & $20.779,9.821,9.593,12.065,19.604,15.418,14.186,19.551,17.584,10.350$ & 12.274 \\
30 & $15.449,9.080,13.102,13.148,13.803,12.591,8.746$ & 11.587 \\
40 & $11.587,11.292,11.218,10.786,13.055$ & 11.540 \\
50 & $9.847,13.752,12.030,10.533$ & 11.765 \\
60 & $8.243,15.787,11.266$ & 10.158 \\
70 & $7.144,11.009,12.321$ & 8.579 \\
80 & $6.452,10.706$ & 8.198 \\
90 & $8.070,8.326$ & 8.507 \\
100 & $7.263,9.751$ & 9.032 \\
110 & 9.032 & 8.688 \\
120 & 8.688 & 8.181 \\
130 & 8.181 & 7.688 \\
140 & 7.688 & 7.175 \\
150 & 7.175 & 6.727 \\
160 & 6.727 & 6.331 \\
170 & 6.331 & \\
\hline
\end{tabular}

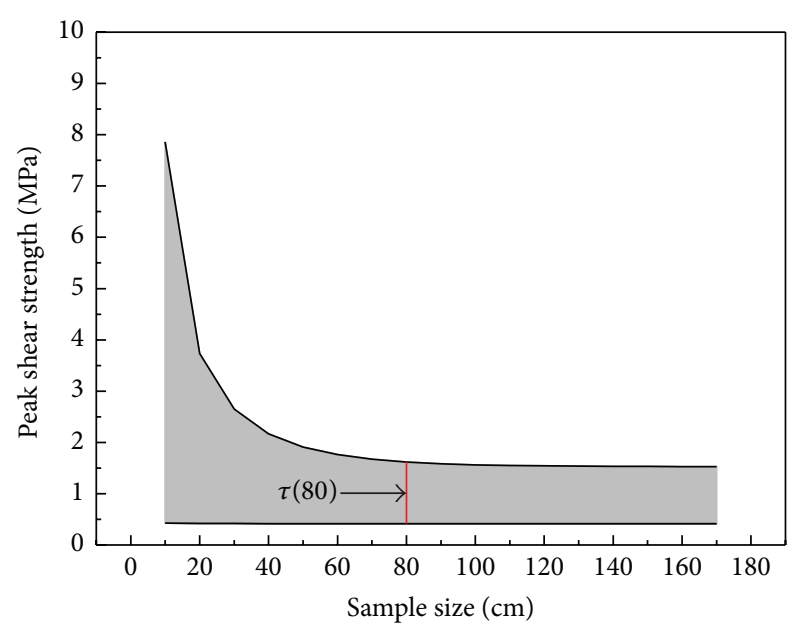

Figure 9: The graph of the neutrosophic function $\tau(L)$ for $L \in[10$, $170]$.

and investigate the neutrosophic functions of the JRC and shear strength of rock joints in different districts in Shaoxing city.

\section{Competing Interests}

The authors declare that they have no competing interests.

\section{Acknowledgments}

This study was funded by the National Natural Science Foundation of China (nos. 41502300, 41427802, 41172292, and
41302257), Zhejiang Provincial Natural Science Foundation (nos. LQ16D020005 and LQ13D020001). The authors appreciate the help provided by Harkiran Kaur, who made a careful English language editing of this paper before submission.

\section{References}

[1] C.-C. Xia, Z.-C. Tang, W.-M. Xiao, and Y.-L. Song, "New peak shear strength criterion of rock joints based on quantified surface description," Rock Mechanics and Rock Engineering, vol. 47, no. 2, pp. 387-400, 2014.

[2] Y.-H. Lee, J. R. Carr, D. J. Barr, and C. J. Haas, "The fractal dimension as a measure of the roughness of rock discontinuity profiles," International Journal of Rock Mechanics and Mining Sciences \& Geomechanics Abstracts, vol. 27, no. 6, pp. 453-464, 1990.

[3] G. Zhang, M. Karakus, H. Tang, Y. Ge, and L. Zhang, "A new method estimating the 2D Joint Roughness Coefficient for discontinuity surfaces in rock masses," International Journal of Rock Mechanics and Mining Sciences, vol. 72, pp. 191-198, 2014.

[4] J.-A. Wang, Y.-X. Wang, Q.-J. Cao, Y. Ju, and L.-T. Mao, "Behavior of microcontacts in rock joints under direct shear creep loading," International Journal of Rock Mechanics and Mining Sciences, vol. 78, pp. 217-229, 2015.

[5] N. Barton, "Review of a new shear-strength criterion for rock joints," Engineering Geology, vol. 7, no. 4, pp. 287-332, 1973.

[6] S. Bandis, A. C. Lumsden, and N. R. Barton, "Experimental studies of scale effects on the shear behaviour of rock joints," International Journal of Rock Mechanics and Mining Sciences and, vol. 18, no. 1, pp. 1-21, 1981.

[7] N. Barton, S. Bandis, and K. Bakhtar, "Strength, deformation and conductivity coupling of rock joints," International Journal of Rock Mechanics and Mining Sciences \& Geomechanics, vol. 22, no. 3, pp. 121-140, 1985. 
[8] Y. Ohnishi, H. Herda, and R. Yoshinaka, "Shear strength scale effect and the geometry of single and repeated rock joints," in Proceedings of 2nd International Conference on Scale Effect in Rock Masses, pp. 167-173, Lisbon, Portugal, 1993.

[9] S. R. Hencher and L. R. Richards, "Assessing the shear strength of rock discontinuities at laboratory and field scales," Rock Mechanics and Rock Engineering, vol. 48, no. 3, pp. 883-905, 2015.

[10] M. J. A. Leal-Gomes and C. Dinis-da-Gama, "Proposal for standardization of pull tests on rock joints," International Journal of Geomechanics, vol. 11, no. 1, pp. 78-82, 2010.

[11] M. Bahaaddini, P. C. Hagan, R. Mitra, and B. K. Hebblewhite, "Scale effect on the shear behaviour of rock joints based on a numerical study," Engineering Geology, vol. 181, pp. 212-223, 2014.

[12] S. Du, Engineering Behavior of Discontinuities in Rock Mass, Seismological Press, Beijing, China, 1999 (Chinese).

[13] S. Chen, W. Zhu, S. Liu, F. Zhang, and L. Guo, "Anisotropy and size effects of surface roughness of rock joints," Chinese Journal of Rock Mechanics and Engineering, vol. 34, no. 1, pp. 57-66, 2015 (Chinese).

[14] N. Barton and S. Bandis, "Review of predictive capabilities of JRC-JCS model in engineering practice," in Proceedings of the International Symposium on Rock Joints, pp. 603-613, Loen, Norway, 1990.

[15] F. Smarandache, Neutrosophic Precalculus and Neutrosophic Calculus, EuropaNova, Brussels, Belgium, 2015.

[16] S. Du, "Research on complexity of surface undulating shapes of rock joints," Journal of China University of Geosciences, vol. 9, no. 1, pp. 86-89, 1998.

[17] N. Barton, "Shear strength investigations for surface mining," in Proceedings of the 23rd US Rock Mechanics Symposium, pp. 178-180, Berkeley, Calif, USA, August 1982.

[18] S. Du, Y. Hu, and X. Hu, "Measurement of joint roughness coefficient by using profilograph and roughness ruler," Journal of Earth Science, vol. 20, no. 5, pp. 890-896, 2009.

[19] G. L. Morelli, "On joint roughness: measurements and use in rock mass characterization," Geotechnical and Geological Engineering, vol. 32, no. 2, pp. 345-362, 2014.

[20] N. Barton and S. Bandis, "Effects of block size on the shear behavior of jointed rock," in Proceedings of the 23rd US Rock Mechanics Symposium, pp. 739-760, Berkeley, Calif, USA, August 1982. 


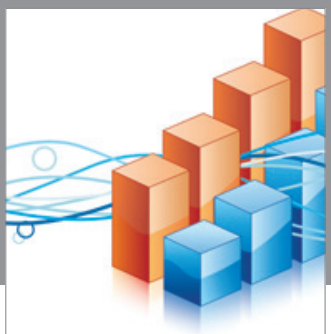

Advances in

Operations Research

vatem alat4

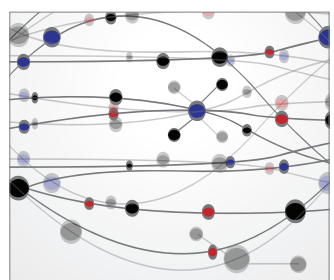

\section{The Scientific} World Journal
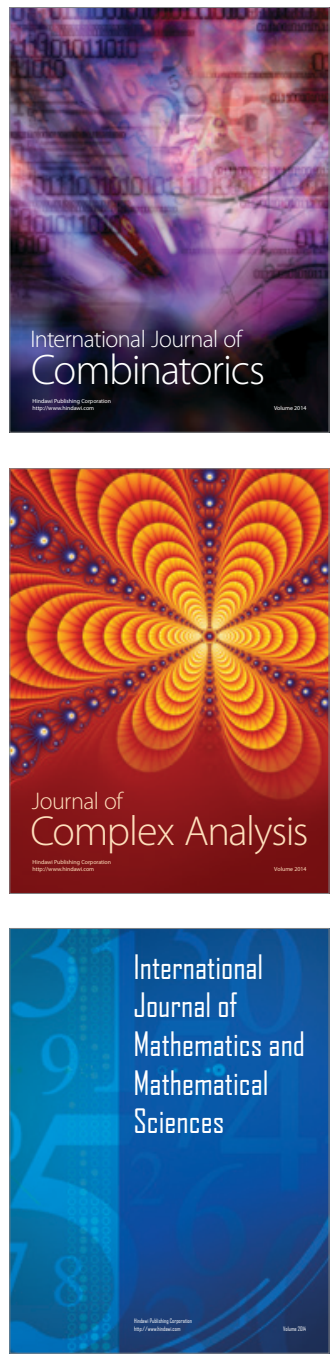
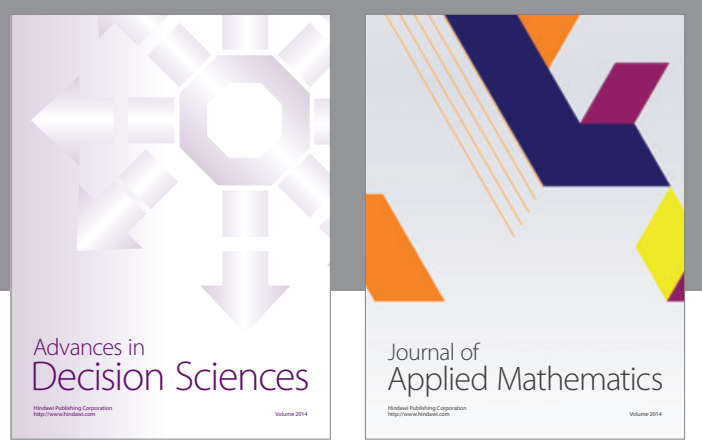

Algebra

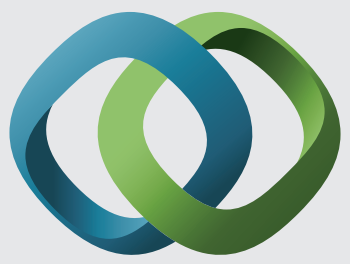

\section{Hindawi}

Submit your manuscripts at

http://www.hindawi.com
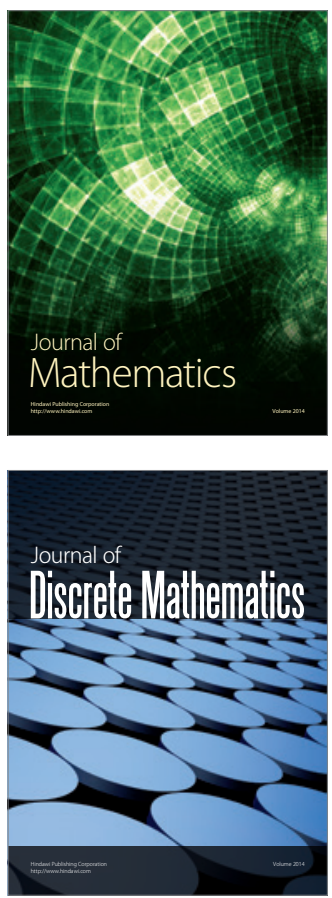

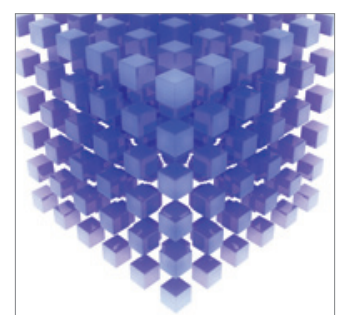

Mathematical Problems in Engineering
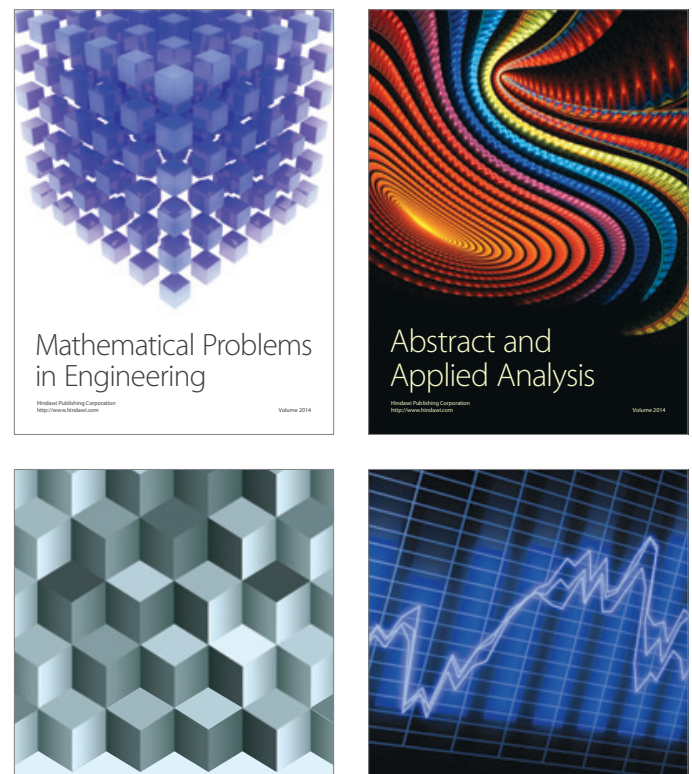

Journal of

Function Spaces

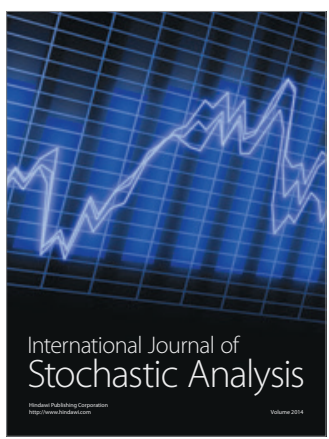

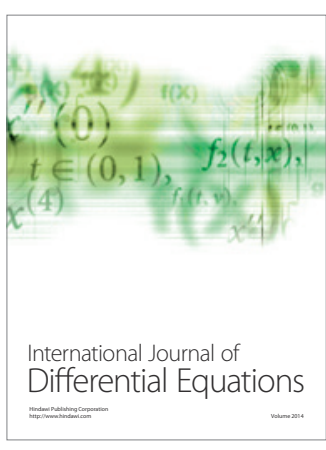
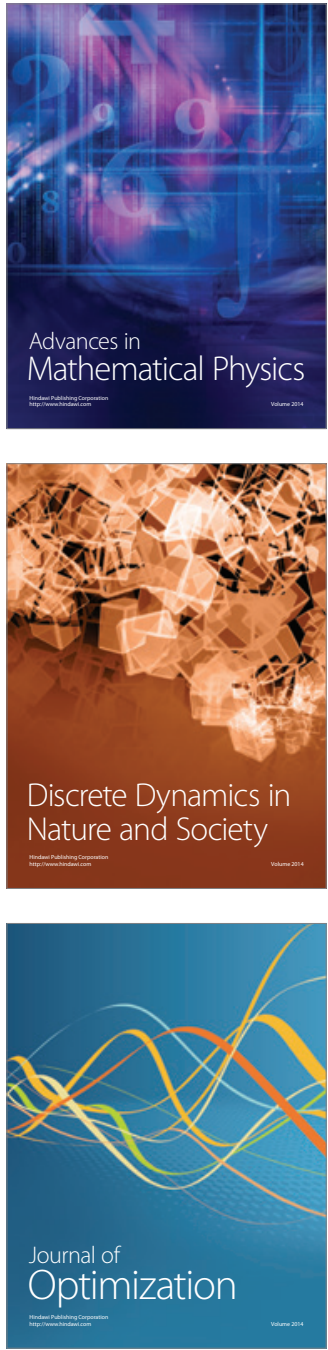